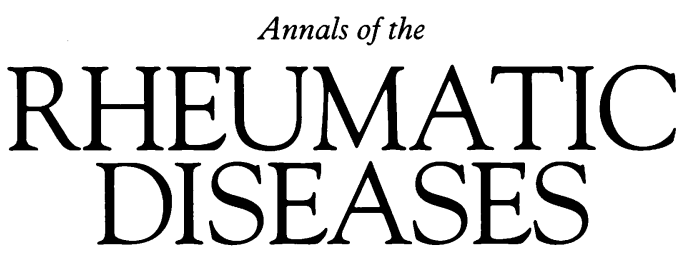

\title{
Adhesion molecules in rheumatoid arthritis: role in the pathogenesis and prospects for therapy
}

Adhesion molecules play a major role in chronic inflammatory conditions such as rheumatoid arthritis (RA). The rheumatoid synovitis is characterised by a mononuclear cell (MNC) infiltrate consisting of clusters of strongly HLA-DR positive antigen presenting cells in close contact with $\mathrm{T}$ lymphocytes, ${ }^{1}$ the majority of which express the helper/memory phenotype (CD4 + CD45R0+). ${ }^{2}$ These immunoreactive foci are thought to be the pathogenetic units responsible for the initiation and perpetuation of RA. This inflammatory infiltrate is generated by a series of events which include the migration of leucocytes from the blood stream into the tissues, their activation to effector cells and, finally, their retention locally to facilitate the ongoing immune reaction. Adhesion molecules are involved in all these phases: first, they mediate the interaction of leucocyte with vascular endothelial cells (EC) during the phenomenon of migration; second, they aid cell contact and deliver costimulatory signals during $\mathrm{Ag}$ presentation and cell activation and third, they allow leucocyte adhesion to extracellular matrix components for local retention.

The first step of leucocyte migration is represented by adhesion to vascular endothelium which has been shown to be a complex multistep process. ${ }^{3}$ In the first instance, leucocytes attach to the EC in the post-capillary venules by means of selectin molecules which bind to their oligosaccharide ligands. Following activation by cytokines released during inflammation, stronger bonds are formed using the $\beta 1$ (VLA-4) and $\beta 2$ (LFA-1 and MAC-1) integrins which bind to their Ig superfamily counterreceptors, VCAM-1 and ICAM-1 respectively. ${ }^{4}$ This more stable adhesion allows transendothelial migration resulting in cell extravasation into the inflamed tissues. The increased ability of CD45R0+ T lymphocytes to bind to EC in vitro and to migrate into epidermal suction blisters in vivo may explain the predominance of these cells at sites of chronic inflammation. ${ }^{5}$ Adhesion molecules also play a major role in the second phase or homotypic/heterotypic cell clustering which is responsible for the formation of the characteristic perivascular MNC aggregates typical of the RA synovium. This process provides the necessary physical conditions for $\mathrm{Ag}$ presentation/response and other vital immune functions requiring cell contact. It is primarily dependent on two adhesion receptor/counter-receptor pairs: LFA-1/ICAM-1 and CD2/LFA3. In the third phase, adhesion molecules are critically important for the binding of inflammatory cells to extracellular matrix components (ECM) such as fibronectin (FN) and collagen (COL) via the $\beta 1$ integrins (VLAs). ${ }^{7}$ Adhesion molecules do not merely act as intercellular anchors but, engaged by their natural ligands, either $\mathrm{FN}^{8}$ or purified VCAM-1 or ICAM-1 ${ }^{9}$ transmit costimulatory signals capable of activating immune cells.

Following the process of activation, adhesion molecules are shed from the cell surface and can be measured in soluble form in the serum. These circulating adhesion molecules have been found to be increased in several autoimmune diseases including RA, SLE and vasculitis. ${ }^{10}$ Although in most cases there is little relation between soluble adhesion molecule levels and disease activity, ${ }^{10}$ in RA a correlation between circulating VCAM-1, but not ICAM-1, has been shown. ${ }^{11}$ The relevance of these soluble forms in disease pathogenesis or the regulation of adhesion remains to be determined; it is possible, however, that these molecules can act as inhibitors of adhesion processes. ${ }^{10}$ In this respect, it is also interesting that some acute phase proteins, such as the $\alpha 1$ acid glycoprotein (orosomucoid), may inhibit selectin mediated leucocyte endothelial interactions. ${ }^{12}$

The regulation of adhesion mechanisms therefore is likely to have an important therapeutic role in the treatment of RA. There has been a renewed interest in studying the effects of well known therapeutic agents on these processes; in addition, new modalities have been developed.

\section{Effects of known therapeutic agents on adhesion related phenomena}

Corticosteroids (CS) are known to induce neutrophilia with lymphopenia in the blood but produce a concomitant decrease of both leucocyte types in inflamed tissues such as the RA synovium. ${ }^{13}$ The mechanism by which this occurs is not completely clear, although interference with the phenomena of migration and recirculation seems an attractive possibility. Support for this idea comes from recent in vitro studies which demonstrate that CS are able to inhibit neutrophil (PMN) EC binding by inhibiting the expression of ELAM-1 and ICAM-1 molecules on EC. ${ }^{14}$ As far as lymphocytes are concerned, CS down regulate the expression of LFA-1 and CD2 following activation, with a consequent decrease in adhesion to endothelium. ${ }^{15}$ 
Colchicine and methotrexate (MTX) have been shown to decrease in vivo leucocyte adhesion and migration in rat mesenteric venules stimulated with platelet activating factor (PAF). ${ }^{16}$ This effect is related to the ability of MTX to increase adenosine release since adenosine inhibits the production of superoxide which is known to increase leucocyte-EC adhesion. ${ }^{17}$ Sulphasalazine, but not sulphapyridine (inactive moiety), has been shown to inhibit the activation-dependent upregulation of CD11b/CD18 (MAC-1) by granulocytes and monocytes but not lymphocytes. ${ }^{18}$ Gold treatment decreases the expression of ELAM-1 by synovial EC in patients with RA. ${ }^{19}$ Finally, in patients with psoriasis, cyclosporin A (CyA) has been shown to reduce dramatically the number of $T$ cells infiltrating the skin and the expression of ICAM-1 on keratinocytes although, interestingly, not on EC. ${ }^{20}$

\section{New therapeutic modalities}

The therapeutic potential of mABs against adhesion molecules has been demonstrated in a variety of animal models including allograft rejection, cardiac reperfusion injury and experimental autoimmune encephalomyelitis. ${ }^{21-23}$ As far as arthritis is concerned, blocking either the LFA-1 or the VLA-4 pathways has been shown to inhibit disease in animals. ${ }^{24}{ }^{25}$ As a result of these studies, a trial using anti ICAM-1 mAb has been conducted in RA. ${ }^{26}$ The patients showed a transient improvement which correlated with the development of a peripheral blood lymphocytosis suggesting that inhibition of lymphocyte migration into inflammatory sites was occurring. However, the therapeutic effect could also have arisen by inhibiting other adhesion-dependent immune functions in which ICAM-1 plays a major role such as $T$ cell activation. Several other ways of modulating cell adhesion are currently being investigated. Of great interest is the use of synthetic oligosaccharides to block the terminal sugars on selectin molecules ${ }^{27}$ and the use of soluble and recombinant ICAM-1 constructs. ${ }^{28}$

\section{Summary}

The essential role played by cell adhesion and migration in the generation of chronic synovitis is now beyond doubt. Some of the beneficial effects of currently used drugs may be related to their ability to interfere with these mechanisms. The complexity of the process, however, means that there are many potential targets for therapeutic intervention and therefore further studies of the basic mechanisms are necessary to identify those which are most important.

Rheumatology Unit,

Guy's Hospital,

London SE1 9RT,

United Kingdom

COSTANTINO PITZALIS

GABRIELLE KINGSLEY GABRIEL PANAYI

Correspondence to: Dr Costantino Pitzalis, Rheumatology Unit, Division of Medicine, Guy's Hospital, St Thomas Street, London SE1 9RT, United Kingdom

1 Janossy G, Panayi G S, Duke O, Bofill M, Poulter L W, Goldstein G. Rheumatoid arthritis: a disease of $\mathrm{T}$ lymphocyte/macrophage immunoregulation. Lancet 1981; ii: $839-42$.

2 Pitzalis C, Kingsley G H, Murphy J, Panayi G S. Abnormal distribution of the helper-inducer and suppressor-inducer $T$ lymphocyte subsets in the rheumatoid joint. Clin Immunol Immunopathol 1987; 45: 252-8.
3 Butcher E C. Leukocyte-endothelial cell recognition: three (or more) steps to specificity and diversity. [Review]. Cell 1991; 67: 1033-6.

4 Springer T A. Adhesion receptors of the immune svistem. Nature 1990; 346: $425-9$.

5 Pitzalis C, Kingsley G H, Haskard D O, Panayi G S. The preferential accumulation of helper-inducer $\mathrm{T}$ lymphocytes in inflammatory lesions: evidence for regulation by selective endothelial and homotypic adhesion. Eur f Immunol 1988; 18: 1397-404.

6 Pitzalis C, Kingsley G H, Covelli M, Meliconi R, Markey A, Panayi G S. Selective migration of the human helper-inducer memory $T$ cell subset: confirmation by in vivo cellular kinetic studies. Eur F Imunol 1991; 21 369-76.

7 Rodriguez R M, Pitzalis C, Kingsley G H, Henderson E M, Humphries M J, Panayi G S. T-lymphocyte adhesion to fibronectin: a possible mechanism for $\mathrm{T}$ cell accumulation in the rheumatoid joint. Clin Exp Immmonol 1992, 89: 439-45.

8 Yamada A, Nikaido T, Noijma Y, Schlossman S F, Morimoto C. Activation of human CD4 T lymphocytes. Interaction of fibronectin with VLA-5 receptor on CD4 cells induces the AP-1 transcription factor. F Immunol 1991; 146: 53-6.

9 Van Seventer G A, Newman W, Shimizu Y, et al. Analysis of T cell stimulation by superantigen plus major histocompatibility complex class II molecules or by $\mathrm{CD} 3$ monoclonal antibody: costimulation by purified adhesion ligands VCAM-1, ICAM-1, but not ELAM-1. I Exp Med 1991 174: $901-13$.

10 Gearing A J H, Newman W. Circulating adhesion molecules in disease. Immunol Today 1993; 14: 506-12.

11 Mason J C, Kapahi P, Haskard D O. Detection of increased levels of circulating intercellular adhesion molecule 1 in some patients with rheumatoid arthritis but not in patients with systemic lupus parients in some patients with adhesion molecule 1. Arthritis Rheum 1993; 36: 519-27.

adhesion molecule 1. Arthritis Rheum 1993; 36: $519-27$ G, van Dijk W. Inflammation-induced expression of sialyl Lewis X-containing glycan structures on alpha 1-acid glycoprotein (orosomucoid) in human sera. f Exp Med 1993; 177: 657-66.

13 Smith M D, Ahern M J, Brooks P M, Roberts-Thomson P J. The clinical and immunological effects of pulse methylprednisolone therapy in rheumatoid arthritis. III. Effects on immune and inflammatory indices in synovial fluid. F Rheum 1988; 15: 238-41.

14 Cronstein B N, Kimmel S C, Levin R I, Martiniuk F, Weissmann G. A mechanism for the antiinflammatory effects of corticosteroids: the glucocorticoid receptor regulates leukocyte adhesion to endothelial cells and expression of endothelial-leukocyte adhesion molecule 1 and intercellular adhesion molecule 1. Proc Nat Acad Sci USA 1992; 89: 9991-5.

15 Pitzalis C, Panayi G S. Inhibition of leucocyte-endothelial interaction, homotypic and heterotypic adhesion by corticosteroids: a possible mechanism for their anti-inflammatory action. Arthritis and Rheum 1993; 36: S246.

16 Asako H, Kubes P, Baethge B A, Wolf R E, Granger D N. Colchicine and methotrexate reduce leukocyte adherence and emigration in rat mesenteric venules. Inflammation 1992; 16: 45-6.

17 Cronstein B N, Eberle M A, Gruber H E, Levin R I. Methotrexate inhibits neutrophil function by stimulating adenosine release from connective tissue cells. Proc Nat Acad USA 1991; 88: 2441-5.

18 Greenfield S M, Hamblin A S, Shakoor Z S, Teare J P, Punchard N A. Inhibition of leucocyte adhesion molecule upregulation by tumor necrosis factor alpha: a novel mechanism of action of sulphasalazine. Gut 1993; 34: 252-6.

19 Corkill M M, Kirkham B W, Haskard D O, Barbatis C, Gibson T, Panayi G S. Gold treatment of rheumatoid arthritis decreases synovial Panayi G S. Gold treatment of rheumatoid arthritis decreases synovial fRheum 1991; 18: 1453-60.

20 Horrocks C, Duncan J I, Oliver A M, Thomson A W. Adhesion molecule expression in psoriatic skin lesions and the influence of cyclosporin A. Clinical and Experimental Immunology 1991; 84: 157-62

21 Isobe $M$, Yagita $\mathrm{H}$, Okamura K, Ihara A. Specific acceptance of cardiac allograft after treatment with antibodies to ICAM-1 and LFA-1. Science 1992; 255: 1125-7.

$22 \mathrm{Ma} \mathrm{X} \mathrm{L}$, Lefer D J, Lefer A M, Rothlein R. Coronary endothelial and cardiac protective effects of a monoclonal antibody to intercellular adhesion molecule-1 in myocardial ischemia and reperfusion. Circulation 1992; 86: 937-46.

23 Yednock T A, Cannon C, Fritz L C, Sanchez-Madrid F, Steinman L, Karin N. Prevention of experimental autoimmune encephalomyelitis by antibodies against alpha 4 beta 1 integrin. Nature 1992; 356: by antibc $63-66$.

24 Jasin H E, Lightfoot E, Davis L S, Rothlein R, Faanes R B, Lipsky P E. Amelioration of antigen-induced arthritis in rabbits treated with monoclonal antibodies to leukocyte adhesion molecules. Arthritis Rheum 1992; 35: 541-9

25 Barbadillo C, Andreu J L, Mulero J, Sanchez-Madrid F. Anti-VLA4 mAb prevents adjuvant arthritis in Lewis rats. Arthritis and Rheum 1993; 36: S95.

26 Kavanaugh A F, Nichols L A, Lipsky P E. Treatment of refractory rheumatoid arthritis with an anti-CD54 (intercellular adhesion molecule-1, ICAM-1) monoclonal antibody. Arthritis and Rheum 1992; 35: $\mathrm{S} 43$.

27 Mulligan M S, Paulson J C, De Frees S, Zheng Z L, Lowe J B, Ward P A. Protective effects of oligosaccharides in P-selectin-dependent lung injury. Nature 1993; 364: 149-51.

28 Martin S, Casasnovas J M, Staunton D E, Springer T A. Efficient neutralization and disruption of rhinovirus by chimeric ICAM-1/ immunoglobulin molecules. fournal of Virologv' 1993; 67: 3561-8. 